\title{
Clima de segurança em Unidades de Terapia Intensiva: Revisão integrativa
}

\author{
Safety climate in Intensive Care Units: Integrative review \\ Clima de seguridad en Unidades de Cuidados Intensivos: Revisión integradora
}

Recebido: 01/06/2021 | Revisado: 07/06/2021 | Aceito: 09/06/2021 | Publicado: 23/06/2021

\author{
Adriana Lemos de Sousa Neto \\ ORCID: https://orcid.org/0000-0002-2389-927X \\ Universidade Federal de Uberlândia, Brasil \\ E-mail: adrianasneto@ufu.br \\ Lara de Andrade Marques \\ ORCID: https://orcid.org/0000-0002-2734-8352 \\ Universidade Federal de Uberlândia, Brasil \\ E-mail: laraenfermagem2011@ hotmail.com \\ Adriana dos Santos Prado Sadoyama \\ ORCID: https://orcid.org/0000-0003-4755-2550 \\ Universidade Federal de Goiás, Brasil \\ E-mail: drisadoyama@gmail.com \\ Geraldo Sadoyama Leal \\ ORCID: https://orcid.org/0000-0003-3594-4194 \\ Universidade Federal de Goiás, Brasil \\ E-mail: gsadoyama@gmail.com \\ Denise Von Dolinger de Brito Röder \\ ORCID: https://orcid.org/0000-0003-4987-3382 \\ Universidade Federal de Uberlândia, Brasil \\ E-mail: denise.roder@ufu.br
}

\begin{abstract}
Resumo
Objetivo: Identificar evidências na literatura sobre o clima de segurança do paciente em unidades de terapia intensiva. Método: Revisão integrativa da literatura, realizada nas bases de dados CINAHL, LILACS, Medline/PubMed, Scopus, Web of Science e na biblioteca Cochrane, para responder a questão norteadora: "Qual o clima de segurança do paciente em unidades de terapia intensiva?”. A busca ocorreu no mês de junho de 2021, onde foram percorridas as seguintes etapas: formulação do problema; busca na literatura dos estudos primários; avaliação dos estudos incluídos na revisão; análise dos dados; apresentação dos resultados. Foram incluídas publicações a qualquer tempo, nos idiomas português, inglês e espanhol. Resultados: Identificou-se 225 referências, das quais 25 foram incluídas na revisão, considerando-se os critérios de inclusão e exclusão. Observou-se, em grande parte da amostra, escores menores que $75 \%$ quanto à percepção do clima de segurança pelos profissionais de saúde avaliados. Conclusão: A identificação de baixos escores na avaliação do clima de segurança do paciente e a fragilidade quanto à notificação dos eventos adversos, apontam a necessidade de maior investimento na cultura de segurança em unidades de terapia intensiva.
\end{abstract}

Palavras-chave: Atitude do pessoal de saúde; Pessoal de saúde; Segurança do paciente; Gestão da segurança; Cuidados críticos; Revisão.

\begin{abstract}
Objective: To identify evidence in the literature about the patient safety climate in intensive care units. Method: Integrative literature review, carried out in the CINAHL, LILACS, Medline / PubMed, Scopus, Web of Science and Cochrane library databases, to answer the guiding question: "What is the patient safety climate in intensive care units?". The search takes place in the months of June 2021, where the following steps were taken: base of the problem; search in the literature of primary studies; evaluation of studies included in the review; data analysis; Results presentation. Publications were published at any time, in Portuguese, English and Spanish. Results: 225 references were identified, of which 25 were included in the review, considering the inclusion and exclusion criteria. In most of the sample, scores below $75 \%$ were observed regarding the perception of the safety climate by the health professionals obtained. Conclusion: The identification of low scores in the assessment of the patient's safety climate and the weakness in reporting adverse events, point to the need for greater investment in the safety culture in intensive care units.
\end{abstract}

Keywords: Attitude of health personnel; Health personnel; Patient safety; Safety management; Critical care; Review.

\section{Resumen}

Objetivo: Identificar evidencia en la literatura sobre el clima de seguridad del paciente en unidades de cuidados intensivos. Método: Revisión integrativa de la literatura, realizada en las bases de datos CINAHL, LILACS, Medline / 
PubMed, Scopus, Web of Science y en la biblioteca Cochrane, para dar respuesta a la pregunta orientadora: “¿Cuál es el clima de seguridad del paciente en las unidades de cuidados intensivos?". La búsqueda se realiza en los meses de junio de 2021, donde se dieron los siguientes pasos: base del problema; búsqueda en la literatura de estudios primarios; evaluación de los estudios incluidos en la revisión; análisis de los datos; Presentación de resultados. Las publicaciones se publicaron en cualquier momento, en portugués, inglés y español. Resultados: se identificaron 225 referencias, de las cuales 25 fueron incluidas en la revisión, considerando los criterios de inclusión y exclusión. Se observó, en gran parte de la muestra, puntuaciones inferiores al $75 \%$ en cuanto a la percepción del clima de seguridad por parte de los profesionales de la salud obtenidos. Conclusión: La identificación de puntuaciones bajas en la evaluación del clima de seguridad del paciente y la debilidad en el reporte de eventos adversos, apuntan a la necesidad de una mayor inversión en la cultura de seguridad en las unidades de cuidados intensivos.

Palabras clave: Actitud del personal de salud; Personel de salud; Seguridad del paciente; Administración de la seguridade; Cuidados críticos; Revisión.

\section{Introdução}

A segurança do paciente, termo amplamente discutido e motivo de preocupação mundial, é considerada um processo multidimensional, que engloba todos os níveis de atenção à saúde, e também multifacetado, por abranger temas como liderança e gestão, ambiente organizacional, recursos humanos, trabalho em equipe, comunicação e envolvimento do paciente e familiares (World Health Organization, 2019).

Entretanto, apesar dos esforços globais na redução dos eventos adversos na assistência à saúde, observados nos últimos 15 anos, mudanças significativas ainda não foram alcançadas (World Health Organization, 2019). Para que haja maior segurança no cuidado prestado, é crucial que uma cultura de segurança seja implantada nas instituições, a qual deve ser constantemente avaliada para possibilitar a identificação de fatores que precisam ser aprimorados dentro do serviço, o direcionamento de ações e a implementação de programas de educação em saúde (Carvalho et al., 2019).

A avaliação da cultura de segurança das organizações pode ser realizada por meio da investigação do clima de segurança, o qual está relacionado à percepção dos profissionais sobre a cultura de segurança nos locais em que atuam, sendo uma ferramenta importante para a análise da mesma (Carvalho et al., 2019). Apesar de os termos clima e cultura serem utilizados de forma intercambiável por muitos pesquisadores, ressalta-se que o clima é o componente mais facilmente mensurável da cultura de segurança, diferente de outros aspectos como comportamento, valores e competências (Sexton et al., 2006).

Ao abordar a segurança do paciente em ambientes hospitalares, um setor que merece destaque é a Unidade de Terapia Intensiva (UTI), isso se justifica pela gravidade do quadro clínico do paciente, pela complexidade e especificidade dos cuidados prestados, a alta tecnologia envolvida e o maior contingente profissional, o que requer investimento na promoção de ações seguras (Duarte et al, 2018).

Considerando que a ocorrência de eventos adversos é uma das 10 principais causas de morte e incapacidade no mundo (Jha, 2018), tendo em vista a necessidade de práticas seguras e a existência de uma cultura de segurança eficaz nos serviços de saúde, em especial nas Unidades de Terapia Intensiva (UTIs), torna-se relevante a realização de estudos que avaliem o clima de segurança dos profissionais que atuam nestas unidades. Assim, esta revisão teve como objetivo identificar evidências na literatura sobre o clima de segurança do paciente em UTIs.

\section{Metodologia}

Trata-se de uma revisão integrativa da literatura em que foram percorridas as seguintes etapas: formulação do problema; busca na literatura dos estudos primários; avaliação dos estudos incluídos na revisão; análise dos dados; apresentação dos resultados (Mendes; Silveira \& Galvão, 2019). 
A busca das publicações ocorreu de forma online nas bases de dados Cumulative Index to Nursing and Allied Health Literature (CINAHL), Biblioteca COCHRANE, Literatura Latino-Americana e do Caribe em Ciências da Saúde (LILACS), Medical Literature and Retrivial System Online (MEDLINE, Pubmed), SCOPUS (Elsevier) e Web of Science (Clarivate Analytics), via portal CAPES, com o uso da plataforma CAFe (Comunidade Acadêmica Federada), no mês de junho de 2021. A população dessa pesquisa foi composta por publicações nacionais e internacionais que abordassem o clima de segurança em unidades de terapia intensiva.

Inicialmente foi estabelecida de forma clara o tema em foco, de modo a facilitar a estratégia de busca, o que resultou na seguinte questão norteadora: Qual o clima de segurança do paciente em UTIs? Como estratégia de busca, foram identificados descritores controlados nos Descritores em Ciências da Saúde (DECs) e Medical Subject Headings (MESH), com posterior combinação dos mesmos por operadores booleanos $O R$ e $A N D$, conforme apresentado no quadro 1. Em algumas bases de dados foi utilizado menor número de descritores para maior recuperação de artigos, visto que não foram encontrados artigos quando combinados mais descritores, como ocorreu na base LILACS.

Quadro 1. Estratégia de busca nas bases de dados. Uberlândia, MG, Brasil, 2021.

\begin{tabular}{|c|c|}
\hline Base de Dados & Estratégia \\
\hline CINAHL & $\begin{array}{l}\text { ("Attitude of Health Personnel" OR "Health Personnel") AND "Patient Safety" AND ("Safety } \\
\text { management" OR "Safety culture") AND ("Critical care" OR "intensive care") }\end{array}$ \\
\hline COCHRANE & $\begin{array}{l}\text { ("Patient Safety" in Title Abstract Keyword AND "Safety management" in Title Abstract Keyword } \\
\text { AND "critical care" in Title Abstract Keyword) }\end{array}$ \\
\hline LILACS & (“segurança do paciente" [Palavras] AND “cuidados críticos" [Palavras]) \\
\hline MEDLINE & $\begin{array}{l}\text { "Attitude of Health Personnel"[All Fields] OR "Health Personnel"[All Fields] AND "Patient } \\
\text { Safety"[All Fields] AND "Safety management"[All Fields] OR "safety culture" AND "Critical } \\
\text { care"[All Fields]) }\end{array}$ \\
\hline SCOPUS & $\begin{array}{l}\text { ((TITLE-ABS-KEY ("health personnel") AND TITLE-ABS-KEY ("Patient Safety") AND TITLE- } \\
\text { ABS-KEY ("Safety management") AND TITLE-ABS-KEY ("Critical care") OR TITLE-ABS- } \\
\text { KEY ("intensive care")) }\end{array}$ \\
\hline Web of Science & $\begin{array}{l}\text { ("attitude of health personnel" OR "health personnel") AND TÓPICO: ("Patient Safety") AND } \\
\text { TÓPICO: ("Safety management") AND TÓPICO: ("critical care") }\end{array}$ \\
\hline
\end{tabular}

Fonte: Autores (2021).

Os critérios de inclusão dos estudos foram: artigos originais; relatos de experiência, protocolos e guidelines disponíveis nas bases de dados online; nos idiomas português, inglês e espanhol; publicações a qualquer tempo; que abordavam o clima de segurança do paciente em UTIs. E os critérios de exclusão: cartas, resumos de anais de eventos, editoriais, livros, teses e dissertações.

Após a recuperação dos artigos conforme os descritores relacionados, utilizou-se a ferramenta de gerenciamento bibliográfico Mendeley ${ }^{\circledR}$ para remoção de artigos duplicados. Realizou-se a leitura do título e resumo do material selecionado, com exclusão daqueles não relacionados à temática proposta e, em seguida, os artigos foram lidos na íntegra, no intuito de identificar informações sobre o clima de segurança do paciente em UTIs. Após aplicados os critérios de inclusão e exclusão, restaram 25 artigos que compuseram a amostra desse estudo.

As informações dos artigos selecionados foram extraídas com a utilização de instrumento baseado nas diretrizes PRISMA, contendo as seguintes informações: autor(es), ano de publicação, área de atuação do primeiro autor, país de origem, tipo de estudo, nível de evidência e instrumento utilizado para avaliar o clima de segurança do paciente.

Os artigos incluídos na revisão foram avaliados quanto ao tipo de estudo e classificados de acordo com os níveis de evidência da Agency for Healthcare Research and Quality (AHRQ): nível 1 - metanálise de múltiplos ensaios clínicos 
controlados e randomizados; nível 2 - estudos individuais com delineamento experimental; nível 3 - estudos quase experimentais; nível 4 - estudos descritivos (não experimentais) ou de abordagem qualitativa; nível 5 - relatos de caso ou experiência; e por fim o nível 6 - opiniões de especialistas (Agency for Healthcare Research and Quality, 2016).

A análise dos dados da revisão foi elaborada de forma descritiva. Foi elaborada tabela síntese com agrupamento dos artigos conforme instrumento utilizado, com divisão dos artigos em duas categorias para melhor visualização dos resultados: Estudos que utilizaram o Safety Attitude Questionnaire (SAQ) na versão adaptada para unidades de cuidado intensivo (SAQICU) e Estudos que utilizaram o Hospital Survey on Patient Safety Culture (HSOPS).

Por se tratar de um artigo de revisão, não foi necessário aprovação em comitê de ética em pesquisa.

\section{Resultados}

A pesquisa nas bases de dados resultou em 225 referências, sendo 111 na MEDLINE, 60 na SCOPUS, 24 na CINAHL, 22 na LILACS, cinco na COCHRANE e três na Web of Science. Foram excluídos 24 artigos repetidos e 91 após a leitura dos títulos e resumos, não relacionados à temática. Assim, restaram 110 para serem lidos na íntegra, dos quais 25 foram incluídos nos resultados desta revisão por abordarem especificamente o tema em questão (Figura 1).

Figura 1. Fluxograma do processo de identificação, seleção e inclusão dos estudos, elaborado a partir da recomendação PRISMA. Uberlândia, MG, Brasil, 2021.
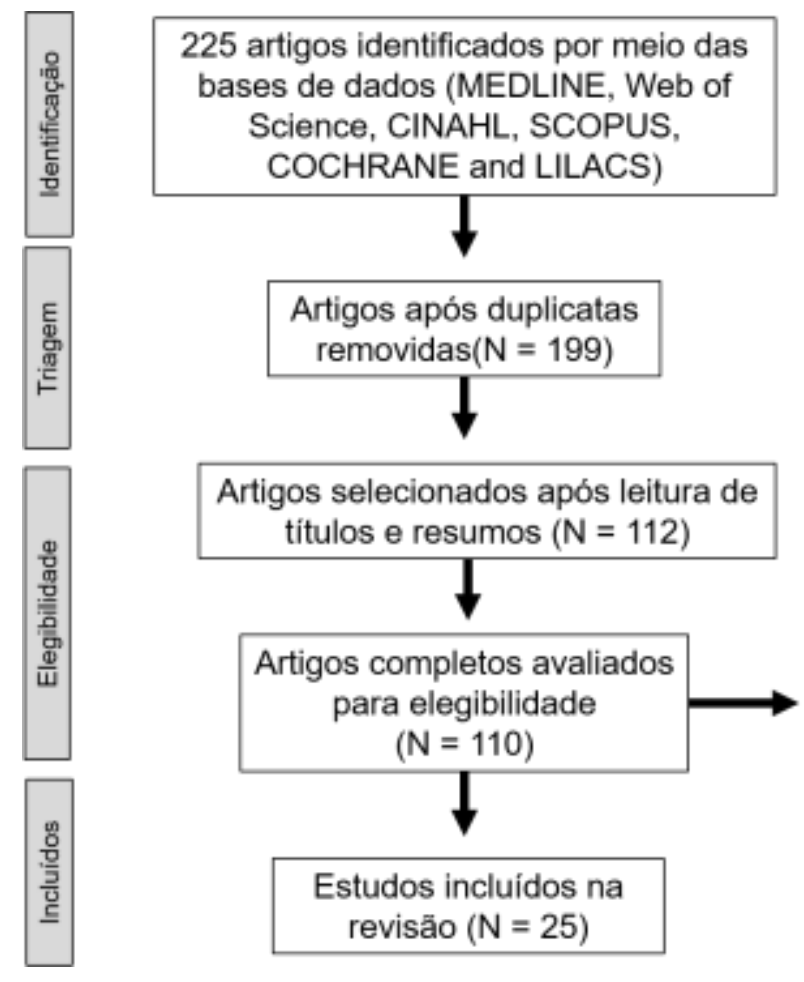

85 artigos de texto completo excluídos:

(06 pela linguagem, 28 artigos de revisão e 51 por não se adequarem ao tema)

Fonte: Autores (2021).

Dentre os países onde foram realizadas as pesquisas, identificou-se predomínio dos Estados Unidos (EUA) com seis trabalhos, seguido do Brasil com quatro e do Irã, Palestina, Arábia Saudita e Chipre, com dois trabalhos cada. Os países Noruega, Holanda, Suécia, Reino Unido, Espanha, Austrália e Tunísia tiveram apenas um estudo cada. 
Os artigos foram publicados entre 2007 e 2020, dos quais 21 (84\%) foram publicados em inglês, três (12\%) em português e um (4\%) em espanhol. De acordo com a AHQR, 21 artigos foram categorizados como nível 4, três nível 3 e um nível 2 (Figura 3). Na maioria dos estudos, 13(52\%), a enfermagem se destacou como área de inserção do primeiro autor.

O Quadro 2 apresenta, em síntese, características gerais e a utilização de instrumento na avaliação do clima de segurança do paciente nos artigos que compuseram a amostra da revisão.

Quadro 2. Caracterização dos estudos selecionados. Uberlândia, MG, Brasil, 2021.

\begin{tabular}{|c|c|c|c|c|}
\hline Citação/Ano/Área de atuação & País & $\begin{array}{c}\text { Tipo } \\
\text { de } \\
\text { Estudo }\end{array}$ & $\begin{array}{c}\text { Nível de } \\
\text { Evidência }\end{array}$ & $\begin{array}{c}\text { Instrumento } \\
\text { utilizado }\end{array}$ \\
\hline Huang et al./2007/Medicina & $\| \mathrm{EUA}$ & $* \mathrm{D}$ & 4 & IISAQ-ICU \\
\hline Gutiérrez-Cía et al. /2010/Medicina & Espanha & $* \mathrm{D}$ & 4 & IISAQ-ICU \\
\hline Armellino, Quinn Griffin \& Fitzpatrick/2010/ Enfermagem & $\| \mathrm{EUA}$ & $* \mathrm{D}$ & 4 & $* *$ HSOPS \\
\hline Sexton et al./2011/Medicina & $\|$ EUA & ${ }^{\dagger} \mathrm{QE}$ & 3 & IISAQ-ICU \\
\hline Poley et al./2011/Medicina & Holanda & $* \mathrm{D}$ & 4 & IISAQ-ICU \\
\hline Profit et al./2012/Medicina & $\| \mathrm{EUA}$ & $* \mathrm{D}$ & 4 & IISAQ-ICU \\
\hline Ballangrud, Hedelin \& Hall-Lord /2012/Enfermagem & Noruega & $* \mathrm{D}$ & 4 & $* *$ HSOPS \\
\hline Meurling et al./2013/Medicina & Suécia & ${ }^{\dagger} \mathrm{QE}$ & 3 & IISAQ-ICU \\
\hline Hamdan, M./2013/Medicina & Palestina & $* \mathrm{D}$ & 4 & IISAQ-ICU \\
\hline Raftopoulos \& Pavlakis/2013/Enfermagem & Chipre & $* \mathrm{D}$ & 4 & IISAQ-ICU \\
\hline Tomazoni et al./2014/Enfermagem & Brasil & $* \mathrm{D}$ & 4 & $* *$ HSOPS \\
\hline Alayed, Lööf \& Johansson/2014/ Biomedicina & $\begin{array}{l}\text { Arábia } \\
\text { Saudita }\end{array}$ & $* \mathrm{D}$ & 4 & IISAQ-ICU \\
\hline Abdi et al./2015/Enfermagem & Irã & $* \mathrm{D}$ & 4 & IISAQ-ICU \\
\hline Livorsi et al./2016/ Medicina & $\| \mathrm{EUA}$ & $* \mathrm{D}$ & 4 & **HSOPS \\
\hline Abu-El-Noor et al./2017/Enfermagem & Palestina & $* \mathrm{D}$ & 4 & IISAQ-ICU \\
\hline Tomazoni et al./2017/Enfermagem & Brasil & $* \mathrm{D}$ & 4 & $* *$ HSOPS \\
\hline Al Malki, Endacott \& Innes /2018/Enfermagem & $\begin{array}{l}\text { Arábia } \\
\text { Saudita }\end{array}$ & $* \mathrm{D}$ & 4 & IISAQ-ICU \\
\hline Amiri, Khademian \& Nikandish 2018/Enfermagem & Irã & ${ }^{\ddagger} \mathrm{ECR}$ & 2 & $* *$ HSOPS \\
\hline Thomas et al./2018/Medicina & $\begin{array}{l}\text { Reino } \\
\text { Unido } \\
\end{array}$ & $* \mathrm{D}$ & 4 & IISAQ-ICU \\
\hline Murphy, Laptook \& Bender /2018/Medicina & $\|$ EUA & ${ }^{\dagger} \mathrm{QE}$ & 3 & $* *$ HSOPS \\
\hline Notaro et al./2019/Enfermagem & Brasil & $* \mathrm{D}$ & 4 & $* *$ HSOPS \\
\hline Souza et al./2019/Enfermagem & Brasil & $* \mathrm{D}$ & 4 & $\S$ \\
\hline Al-Mugheed \& Bayraktar/ 2020/Enfermagem & Chipre & $* \mathrm{D}$ & 4 & IISAQ-ICU \\
\hline Dunstan \& Coyer /2020/Enfermagem & Austrália & $* \mathrm{D}$ & 4 & ISAQ-ICU \\
\hline Tlili et al./2020/Enfermagem & Tunísia & $* \mathrm{D}$ & 4 & $* *$ HSOPS \\
\hline
\end{tabular}

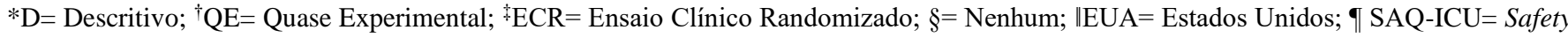
Attitude Questionnaire (SAQ) na versão adaptada para unidades de cuidado intensivo; **HSOPS: Hospital Survey on Patient Safety Culture. Fonte: Autores (2021). 
O instrumento de avaliação do clima de segurança mais utilizado nos artigos foi SAQ-ICU, seguido do HSOPS. A seguir estão apresentados, de forma categorizada por instrumento utilizado, os principais resultados dos estudos (Quadros 3 e $4)$.

Quadro 3. Categoria "Estudos que utilizaram o Safety Attitude Questionnaire, versão adaptada para unidades de cuidado intensivo (SAQ-ICU)" na avaliação do clima de segurança do paciente. Uberlândia, MG, Brasil, 2021.

\begin{tabular}{|c|c|}
\hline Autor & Principais resultados \\
\hline Huang et al. & $\begin{array}{l}\text { Escore geral }<75 \% \text {. Enfermeiros apresentaram escores gerais maiores que médicos. Piores } \\
\text { escores: "condições de trabalho" e "reconhecimento do estresse". Respostas variaram entre as } \\
\text { *UTIs avaliadas. }\end{array}$ \\
\hline Gutiérrez-Cía et al. & $\begin{array}{l}\text { Escore geral < 75\%. Unidades maiores tiveram piores escores. Domínios com menor pontuação: } \\
\text { percepção de gestão e condições de trabalho. }\end{array}$ \\
\hline Sexton et al. & $\begin{array}{l}\text { Melhora nos escores do clima de segurança de } 42,5 \% \text { (2004) para 52,2\% (2006). Cinco dos sete } \\
\text { itens de clima de segurança melhoraram. }\end{array}$ \\
\hline Poley et al. & $\begin{array}{l}\text { Melhora dos escores gerais de } 2006 \text { para 2007. Piora apenas nas pontuações sobre "condições de } \\
\text { trabalho" e "reconhecimento de estresse". }\end{array}$ \\
\hline Profit et al. & $\begin{array}{l}\text { Escore com média de } 86 \% \text {. Enfermagem apresentou escores mais baixos que médicos. Respostas } \\
\text { variaram entre as *UTIs. }\end{array}$ \\
\hline Meurling et al. & $\begin{array}{l}\text { Escore < } 75 \% \text {. Médicos tiveram melhor pontuação antes da intervenção. Enfermeiros tiveram } \\
\text { maior pontuação após intervenção. }\end{array}$ \\
\hline Hamdan, M. & $\begin{array}{l}\text { Pontuações médias de } 71,22 \% \text { para satisfação no trabalho e } 63 \% \text { para "reconhecimento de } \\
\text { estresse"; escores variaram entre as *UTIs. } 71,0 \% \text { não notificaram evento adverso no último ano. }\end{array}$ \\
\hline $\begin{array}{l}\text { Raftopoulos \& } \\
\text { Pavlakis }\end{array}$ & $\begin{array}{l}\text { Menores escores: reconhecimento do estresse. Enfermeiros com mais experiência tiveram maior } \\
\text { pontuação para os itens "trabalho em equipe" e "percepção de gestão" do que os enfermeiros }\end{array}$ \\
\hline $\begin{array}{l}\text { Alayed, Lööf \& } \\
\text { Johansson }\end{array}$ & $\begin{array}{l}\text { Enfermeiros assistenciais tiveram maior pontuação comparados aos demais enfermeiros. A } \\
\text { pontuação mais baixa foi } 78,8 \% \text {, sobre "percepção de gestão". }\end{array}$ \\
\hline Abdi et al. & $\begin{array}{l}\text { Escores }<75 \% \text {. Médicos tiveram escores maiores que enfermeiros. Entrevistas revelaram } \\
\text { subnotificação, baixa satisfação entre os enfermeiros e comunicação ineficaz. }\end{array}$ \\
\hline Abu-El-Noor et al. & $\begin{array}{l}\text { A pontuação geral foi de } 63,9 \% \text {. Escores mais baixos para "percepção de gestão" e "condições de } \\
\text { trabalho". Houve variação entre as *UTIs. }\end{array}$ \\
\hline $\begin{array}{l}\text { Al Malki, Endacott \& } \\
\text { Innes }\end{array}$ & $\begin{array}{l}\text { Escores < 75\% (domínios variaram de 47,1 a 70,3) com a menor pontuação ao domínio } \\
\text { "percepções de gestão" e "condições de trabalho". }\end{array}$ \\
\hline Thomas et al. & $\begin{array}{l}\text { Enfermagem apresentou menores escores. Funcionários mais experientes apresentaram pontuações } \\
\text { médias maiores. }\end{array}$ \\
\hline $\begin{array}{l}\text { Al-Mugheed \& } \\
\text { Bayraktar }\end{array}$ & $\begin{array}{l}\text { Escores gerais }<75 \% \text {. O maior escore foi para o clima de segurança e o pior foi sobre o } \\
\text { reconhecimento de estresse. }\end{array}$ \\
\hline Dunstan \& Coyer & $\begin{array}{l}\text { Escores baixos no domínio de reconhecimento do estresse. Médicos perceberam a dimensão } \\
\text { "condições de trabalho" como mais positivas do que a enfermagem. }\end{array}$ \\
\hline
\end{tabular}

*UTIs: Unidades de Terapia Intensiva.

Fonte: Autores (2021). 
Quadro 4. Categoria "Estudos que utilizaram o Hospital Survey on Patient Safety Culture (HSOPS)” na avaliação do clima de segurança do paciente. Uberlândia, MG, Brasil, 2021.

\begin{tabular}{|c|c|}
\hline Autor & Principais resultados \\
\hline $\begin{array}{l}\text { Armellino, Quinn } \\
\text { Griffin \& Fitzpatrick }\end{array}$ & $\begin{array}{l}\text { A maioria }(62,5 \%) \text { não relatou erro nos últimos } 12 \text { meses. Quanto mais tempo no serviço, menor } \\
\text { percepção positiva do trabalho. }\end{array}$ \\
\hline $\begin{array}{l}\text { Ballangrud, Hedelin } \\
\& \text { Hall-Lord }\end{array}$ & $\begin{array}{l}\text { A média total das pontuações em todas as dimensões foi de } 55 \% \text {. Cerca de } 50 \% \text { dos enfermeiros } \\
\text { não notificaram incidentes. }\end{array}$ \\
\hline Tomazoni et al. & $\begin{array}{l}\text { Maior tempo de trabalho na profissão representou menores escores e menos eventos comunicados. } \\
\text { Médicos tiveram maior pontuação. }\end{array}$ \\
\hline Livorsi et al. & $\begin{array}{l}\text { Pontuação baixa quanto à notificação de eventos adversos. Em entrevista, } 52 \% \text { relataram falha na } \\
\text { comunicação nas mudanças de turno. }\end{array}$ \\
\hline Tomazoni et al. & $\begin{array}{l}\text { Principais discussões: estratégias para segurança do paciente; fatores de risco que interferem na } \\
\text { segurança; dificuldades na comunicação de erros. }\end{array}$ \\
\hline $\begin{array}{l}\text { Amiri, Khademian \& } \\
\text { Nikandish }\end{array}$ & $\begin{array}{l}\text { O grupo experimental obteve escores médios totais pós-intervenção superiores aos do grupo } \\
\text { controle. Melhora em sete das dez dimensões avaliadas. }\end{array}$ \\
\hline $\begin{array}{l}\text { Murphy, Laptook \& } \\
\text { Bender }\end{array}$ & A intervenção de 12 meses melhorou a maioria das atitudes de segurança da equipe. \\
\hline Notaro et al. & $\begin{array}{l}\text { A dimensão "Resposta não punitiva ao erro" teve menor pontuação. Enfermeiros foram os que mais } \\
\text { notificaram eventos adversos. }\end{array}$ \\
\hline Tlili et al. & $\begin{array}{l}\text { Todas as dimensões tiveram pontuação baixa. A dimensão com maior pontuação foi "trabalho em } \\
\text { equipe" }(47,87 \%) \text { e a menor foi "resposta não punitiva ao erro" }(18,6 \%) \text {. }\end{array}$ \\
\hline
\end{tabular}

Fonte: Autores (2021).

Apenas um artigo não utilizou instrumento na avaliação do clima de segurança. Trata-se de um estudo qualitativo, onde foram realizadas entrevistas que revelaram falta de comunicação e notificação de eventos adversos. A sobrecarga de trabalho e fadiga foram apontados como fatores contribuintes para o cuidado não seguro (Souza et al, 2019).

Quatro artigos apresentaram resultado de intervenções educacionais que objetivaram melhorar o clima de segurança do paciente em UITs (Sexton et al, 2011; Meurling et al, 2013; Amiri, Khademian \& Nikandish, 2018; Murphy, Laptook \& Bender, 2018).

\section{Discussão}

Os baixos escores de pontuação quanto à percepção geral do clima de segurança, encontrados em vários artigos, refletem a necessidade de fortalecimento da cultura de segurança das UTIs avaliadas, motivo pelo qual é essencial a divulgação dos resultados de pesquisas sobre o clima de segurança do paciente aos gestores das instituições envolvidas.

Destaca-se a predominância da utilização dos questionários Safety Attitude Questionnaire (SAQ-ICU) e Hospital Survey on Patient Safety Culture (HSOPS) na avaliação do clima de segurança nos estudos avaliados, escolha que pode ter sido atribuída ao fato de serem instrumentos validados e traduzidos em vários idiomas. Desenvolvidos para profissionais que trabalham em hospitais e têm ou não contato direto com o paciente, mas cujas funções executadas afetam diretamente o cuidado destinado ao mesmo, os instrumentos citados foram elaborados com o objetivo de mensurar múltiplas dimensões da cultura de segurança do paciente e verificam, junto aos seus respondentes, questões importantes relacionadas à segurança valores, crenças e normas da organização, notificação/relato de eventos adversos, comunicação, liderança e gestão (Sousa \& Mendes, 2019). Ambos são amplamente citados em pesquisas de todo o mundo que objetivam avaliar a cultura de segurança do paciente em ambientes hospitalares (Okuyama et al., 2019). 
O HSOPS, desenvolvido pela AHRQ em 2004, é composto em sua versão original por 42 itens distribuídos em 12 dimensões para avaliar a cultura do paciente na perspectiva dos profissionais em ambiente hospitalar (Sorra \& Nieva, 2004).

Oriundo da Universidade do Texas, o SAQ é um instrumento validado em 60 itens distribuídos em seis dimensões, que estima as percepções dos profissionais de saúde sobre o clima de segurança e considera positivas respostas superiores a 75\% (Sexton et al, 2006). Assim como o HSOPS, os itens são respondidos usando uma escala tipo Likert de cinco pontos, cujos extremos são "discordo totalmente" a "concordo totalmente". O SAQ-ICU possui o mesmo conteúdo da versão genérica, com pequenas modificações que refletem as unidades críticas, como o nível de estresse e ambiente de trabalho, características relevantes em UTIs (Sexton et al, 2006, Gutiérrez-Cía et al, 2010).

Uma evidência comum em alguns estudos foram os melhores escores gerais dos médicos comparados aos escores dos enfermeiros, o que pode ser atribuído ao estresse e desafios decorrentes da escassez de pessoal e das condições complexas que envolvem a assistência de enfermagem aos pacientes em cuidados críticos, influenciando diretamente a pontuação da enfermagem quando da aplicação dos instrumentos que avaliam o clima de segurança (Huang, Wu \& Lee, 2018). As percepções discrepantes entre médicos e enfermeiros na avaliação do trabalho em equipe e satisfação no trabalho podem estar relacionadas às diferentes responsabilidades, culturas e status entre as duas categorias profissionais (Thomas et al, 2003).

Condições de trabalho e reconhecimento do estresse foram dimensões que apresentaram baixas pontuações em muitos estudos, principalmente pela enfermagem. Na linha de frente na assistência à saúde, a equipe de enfermagem é a primeira a identificar possíveis falhas, além de lidar constantemente com sobrecarga de trabalho, deficiente infraestrutura, inadequação de recursos humanos e número elevado de pacientes (Carvalho et al, 2019; Sousa \& Mendes, 2019). Nesse ínterim, faz-se necessário a disponibilização de recursos adequados, seja com políticas ou práticas que promovam melhores condições de trabalho, uma gestão que fomente uma comunicação adequada e valorização dos profissionais, os quais podem contribuir positivamente na tomada de decisões em prol da qualidade do cuidado prestado (Carvalho et al, 2019; Al-Mugheed K \& Bayraktar, 2020).

O processo de trabalho em saúde é complexo e passível de erros/falhas humanas. No entanto, a ausência desse tema na formação dos profissionais de saúde, associada à falta de uma cultura de apoio institucional e dos pares, fazem com que ainda seja difícil realizar a comunicação e notificação de um evento adverso (Sousa \& Mendes, 2019). Não obstante, os achados desta revisão apontaram fragilidade na comunicação e notificação de eventos adversos e no manejo do erro, sendo a mudança de cultura e estímulo aos profissionais no reconhecimento de suas falhas, estratégias essenciais para a promoção de práticas seguras, o que propiciará elaboração de ações voltadas à implantação de barreiras de segurança nos serviços de saúde (Gutiérrez-Cía et al, 2010; Ballangrud, Hedelin \& Hall-Lord, 2012; Tomazoni et al, 2014; Abdi et al, 2015; Tomazoni et al, 2017, Notaro et al, 2019; Souza et al, 2019; Pavan et al, 2019, Moreno \& Andreoli, 2019).

Percepções e atitudes mais positivas podem ser identificadas nos profissionais após mudanças organizacionais associadas a intervenções, o que foi evidenciado em quatro artigos dessa revisão, os quais apresentaram resultado de processo educacional no clima de segurança das equipes de saúde que atuam nas UTIs avaliadas. Os programas educacionais em saúde possibilitam um aumento da confiança dos profissionais, maior valorização e melhoria da comunicação e do trabalho em equipe (Meurling et al, 2013; Milton et al, 2020). Sabe-se ainda, que a educação permanente em saúde tem contribuído para a transformação das práticas dos profissionais de saúde, pois a mesma reconhece a capacidade da pessoa de interagir com o outro e com os cenários da vida e da saúde, oportunizando possibilidades de troca de conhecimentos, culminando na construção de saberes significativos nos espaços de saúde (Rojas et al, 2019).

Em estudos que avaliaram mais de uma UTI, foi observado uma variedade significativa de escores gerais sobre o clima de segurança entre as unidades, resultados que reafirmam a importância de implementação de programas educacionais 
específicos, elaborados conforme a realidade individual e diversidade de demandas de cada unidade hospitalar (Gutiérrez-Cía et al, 2010; Profit et al, 2012, Hamdan, 2013; Abu-El-Noor et al, 2017).

A realização de pesquisas com bom nível de evidência, que busquem soluções efetivas com vistas à práticas seguras em saúde e com redução do dano ao paciente, são relevantes e necessárias (Sousa \& Mendes, 2019). Entretanto, a estratégia de buscas dessa revisão resultou em uma amostra composta, em sua maior parte, por estudos descritivos, o que pode ser considerada uma limitação do estudo. Sugere-se, assim, a realização de novos estudos de intervenção em UTIs, com enfoque na melhoria do clima de segurança, o que culminará em um cuidado mais seguro e de maior qualidade aos usuários dos serviços de saúde.

Essa revisão contribuirá com a literatura científica ao apresentar evidências sobre o clima de segurança do paciente em UTIs, que reflete não apenas as percepções e atitudes de profissionais de saúde, mas também lacunas na cultura de segurança desses setores, instiga gestores e todos os envolvidos na assistência à saúde sobre a necessidade de maiores investimentos em condições de trabalho adequadas, reconhecimento de falhas na comunicação, manejo correto de eventos adversos, revisão das políticas de saúde e implantação de intervenções educacionais. Tais avanços beneficiarão, certamente, aqueles que dão sentido aos estudos e a todas as ações dos serviços de saúde: os pacientes necessitados de cuidados intensivos, seguros e de qualidade.

\section{Conclusão}

Muitos são os desafios quando o assunto é a segurança do paciente. A identificação de baixos escores na avaliação do clima de segurança do paciente e falhas no processo de comunicação e notificação de eventos adversos apontam que deve haver maior investimento na cultura de segurança em UTIs.

Destaca-se, nesse sentido, o papel dos gestores em fomentar processos de trabalho que envolvam a equipe interprofissional no sentido criar estratégias voltadas às práticas seguras e reconhecimento de entraves a serem solucionados, além de viabilizar o desenvolvimento de intervenções e processos educacionais, caminho principal para a consolidação de uma cultura de segurança.

\section{Referências}

Abdi Z., Delgoshaei B., Ravaghi H., Abbasi M. \& Heyrani A. (2015). The culture of patient safety in an Iranian intensive care unit. J Nurs Manag. 23(3):33345. 10.1111/jonm.12135

Abu-El-Noor N. I., Hamdan M. A., Abu-El-Noor M. K., Radwan A-K. S. \& Alshaer A. A. (2017). Safety Culture in Neonatal Intensive Care Units in the Gaza Strip, Palestine: A Need for Policy Change. J Pediatr Nurs. 33:76-82. 10.1016/j.pedn.2016.12.016

Alayed A. S. , Lööf H. \& Johansson U-B. (2014). Saudi Arabian ICU safety culture and nurses’ attitudes. Int J Health Care Qual Assur. ;27(7):581-93. 10.1108/IJHCQA-04-2013-0042

Agency for Healthcare Research and Quality (AHRQ). (2016). Quality Improvement and monitoring at your fingertips. Rockville. Available from: https://qualityindicators.ahrq.gov/

Al Malki A., Endacott R. \& Innes K. (2018). Health professional perspectives of patient safety issues in intensive care units in Saudi Arabia. J Nurs Manag. 26(2):209-18. 10.1111/jonm.12536

Al-Mugheed K. \& Bayraktar N. (2020). Patient safety attitudes among critical care nurses: A case study in North Cyprus. Int J Health Plann Manage. $10.1002 / \mathrm{hpm} .2976$

Amiri M., Khademian Z. \& Nikandish R. (2018). The effect of nurse empowerment educational program on patient safety culture: a randomized controlled trial. BMC Med Educ. 18(1):158. 10.1186/s12909-018-1255-6

Armellino D., Quinn Griffin M. T. \& Fitzpatrick JJ. (2010). Structural empowerment and patient safety culture among registered nurses working in adult critical care units. J Nurs Manag. 18(7):796-803. 10.1111/j.1365-2834.2010.01130.x

Ballangrud R, Hedelin B \& Hall-Lord M. L.(2012). Nurses' perceptions of patient safety climate in intensive care units: A cross-sectional study. Intensive Crit Care Nurs. 28(6):344-54. 10.1016/j.iccn.2012.01.001 
Carvalho P. A., Albina C., Laundos S., Ventura J. \& Juliano S. (2019). Assessment of safety culture in a public hospital in the Federal District, Brazil. Heal Serv Assess [Internet]. 72(Suppl 1):263-9. Available from: http://dx.doi.org/10.1590/0034-7167-2017-0716

Duarte S. da C. M., Stipp M. A. C., Cardoso M. M. V. N. \& Büscher A. (2018). Patient safety: understanding human error in intensive nursing care. Rev da Esc Enferm da USP [Internet].52(e03406):1-8. Available from: http://dx.doi.org/10.1590/S1980-220X2017042203406

Dunstan E. \& Coyer F. (2020). Safety culture in two metropolitan Australian tertiary hospital intensive care units: A cross-sectional survey. Aust Crit Care. 33(1):4-11. 10.1016/j.aucc.2018.11.069

Gutiérrez-Cía I., De Cos P. M., Juan A. Y., Obón-Azuara B., Alonso-Ovies A., Martin-Delgado M. C., Álvarez-Rodríguez J., \& Aibar-Remón C. (2010). Perception of safety culture in Spanish intensive care units | Percepción de la cultura de seguridad en los servicios de medicina intensiva españoles. Med Clin (Barc). 135(SUPPL.):37-44. 10.1016/S0025-7753(10)70019-1

Hamdan M. (2013). Measuring safety culture in Palestinian neonatal intensive care units using the Safety Attitudes Questionnaire. J Crit Care. 28(5):886.e7886.e14. 10.1016/j.jcrc.2013.06.002

Huang D. T., Clermont G., Sexton J. B., Karlo C. A., Miller R. G., Weissfeld L. A., Rowan K. M., \& Angus D. C. (2007). Perceptions of safety culture vary across the intensive care units of a single institution. Crit Care Med. 35(1):165-76. 10.1097/01.CCM.0000251505.76026.CF

Huang C-H., Wu H- \& Lee Y-C. (2018). The perceptions of patient safety culture: A difference between physicians and nurses in Taiwan. Appl Nurs Res [Internet]. 40:39-44. 10.1016/j.apnr.2017.12.010. Available from: http://www.sciencedirect.com/science/article/pii/S0897189716300775

Jha A. K. (2018). Presentation at the "Patient Safety - A Grand Challenge for Healthcare Professionals and Policymakers Alike" a Roundtable at the Grand Challenges Meeting of the Bill \& Melinda Gates Foundation. Available from: https://globalhealth.harvard.edu/qualitypowerpoin

Livorsi D., Knobloch M. J., Blue L. A., Swafford K., Maze L., Riggins K., Hayward T., \& Safdar N. (2016). A rapid assessment of barriers and facilitators to safety culture in an intensive care unit. Int Nurs Rev. 63(3):372-6. 10.1111/inr.12254

Mendes K. D. S., Silveira R. C. C. P. \& Galvão C. M. (2019). Revisão integrativa: método de pesquisa para a incorporação de evidências na saúde e na enfermagem. Texto Contexto Enferm. 17(4): 758-64. Available from: https://www.scielo.br/j/tce/a/XzFkq6tjWs4wHNqNjKJLkXQ/?lang=pt\&format=pdf.

Meurling L., Hedman L., Sandahl C., Fellander-Tsai L .\& Wallin C-J. (2013). Systematic simulation-based team training in a Swedish intensive care unit: a diverse response among critical care professions. BMJ Qual Saf. 22(6):485-94. 10.1136/bmjqs-2012-000994

Milton J., Chaboyer W., Åberg N. D., Erichsen Andersson A. \& Oxelmark L. (2020). Safety attitudes and working climate after organizational change in a major emergency department in Sweden. Int Emerg Nurs [Internet]. 100830. 10.1016/j.ienj.2020.100830. Available from: http://www.sciencedirect.com/science/article/pii/S1755599X2030001X

Moreno M. C. \& Andreoli P. de A. (2019). Segurança do paciente: não é uma intenção, é uma ação. In: Bopsin P dos S, Ribas EO, Silva DM, editors. Guia Prático para Segurança do Paciente. Porto Alegre: Moriá; p. 255.

Murphy T., Laptook A. \& Bender J. (2018). Sustained Improvement in Neonatal Intensive Care Unit Safety Attitudes After Teamwork Training. J Patient Saf. 14(3):174-80.

Notaro K. A. M., Corrêa A. R., Tomazoni A., Rocha P. K. \& Manzo B. F. (2019). Safety culture of multidisciplinary teams from neonatal intensive care units of public hospitals. Rev Lat Am Enfermagem. 27. 10.1590/1518-8345.2849-3167

Okuyama J. H. H., Galvão T. F., Crozatti M. T. L., Silva M. T. (2019). Health professionals' perception of patient safety culture in a university hospital in São Paulo: A cross-sectional study applying the Hospital Survey on Patient Safety Culture. Sao Paulo Med J [Internet]. May [cited 2020 Jul 22];137(3):216-22. 10.1590/1516-3180.2018.0430140319. Available from: http://www.scielo.br/scielo.php?script=sci_arttext\&pid=S1516-31802019000300216\&tlng=en

Pavan N. F. P., Magalhães A. L. P., Poncio D. F. , Ascari R. A., Zanini P. D. , Knihs N. da S, Silva O. M. (2019). Patient safety culture in kidney transplant patients in western Santa Catarina. Acta Paul Enferm [Internet]. Aug [cited 2020 Jun 8];32(4):398-405. 10.1590/1982-0194201900055. Available from: http://www.scielo.br/scielo.php?script=sci_arttext\&pid=S0103-21002019000400398\&tlng=pt

Poley M. J., van der Starre C., van den Bos A., van Dijk M. \& Tibboel D. (2011). Patient safety culture in a Dutch pediatric surgical intensive care unit: an evaluation using the Safety Attitudes Questionnaire. Pediatr Crit Care Med. 12(6):e310-6. 10.1097/PCC.0b013e318220afca

Profit J., Etchegaray J., Petersen L.A., Sexton J. B., Hysong S. J., Mei M., \& Thomas E. J. (2012). Neonatal intensive care unit safety culture varies widely. Arch Dis Child Fetal Neonatal Ed. 97(2). 10.1136/archdischild-2011-300635

Raftopoulos V. \& Pavlakis A. (2013). Safety climate in 5 intensive care units: a nationwide hospital survey using the Greek-Cypriot version of the safety attitudes questionnaire. J Crit Care. Feb;28(1):51-61. 10.1016/j.jcrc.2012.04.013

Rojas F. L. L., Kehrig R. T., Biato E. C. L. \& Santos N. C. dos. (2019). Permanent education in health: the rethinking about the construction of heath practices. J Heal NPEPS [Internet].4(2):310-30. 10.30681/252610103730. Available from: https://periodicos.unemat.br/index.php/jhnpeps/article/view/3730/3362

Sexton J. B., Berenholtz S. M., Goeschel C. A., Watson S. R., Holzmueller C. G., Thompson D. A., Hyzy R. C., Marsteller J. A., Schumacher K., \& Pronovost P. J. (2011). Assessing and improving safety climate in a large cohort of intensive care units. Crit Care Med [Internet]. 39(5):934-9. 10.1097/CCM.0b013e318206d26c. Available from: http://search.ebscohost.com/login.aspx ?direct=true\&db=c8h\&AN=104710839\&lang=pt-br\&site=ehostlive\&authtype=ip,cookie,uid

Sexton J. B., Helmreich R. L., Neilands T. B., Rowan K., Vella K., Boyden J., Roberts P. R., \& Thomas, E. J. (2006). The Safety Attitudes Questionnaire: psychometric properties, benchmarking data, and emerging research. BMC Health Serv Res. 6:44.10.1186/1472-6963-6-44

Sorra J. S. \& Nieva V. F. (2004). Hospital Survey on Patient Safety Culture. (Prepared by Westat, under Contract No 290-96-0004) AHRQ Publication no 040041 [Internet]. 2004; Available from: https://proqualis.net/sites/proqualis.net/files/User guide HSOPSC.pdf . 
Research, Society and Development, v. 10, n. 7, e33910716702, 2021

(CC BY 4.0) | ISSN 2525-3409 | DOI: http://dx.doi.org/10.33448/rsd-v10i7.16702

Sousa, P. \& Mendes, W. (2019). Segurança do paciente: criando organizações de saúde seguras. 2 edição. FIOCRUZ, editor. Rio de Janeiro: CDEAD; 268 p.

Souza C. S. de, Tomaschewski-Barlem J. G., Rocha L. P., Barlem E. L. D., Silva T. L. \& Neutzling B. R. da S. (2019). Patient safety culture in intensive care units: perspective of health professionals. Rev Gauch Enferm. 40(spe):e20180294. 10.1590/1983-1447.2019.20180294

Thomas A. \& Lomas J-P. (2018). Establishing the use of a safety attitudes questionnaire to assess the safety climate across a critical care network. J Intensive Care Soc. Aug;19(3):219-25. 10.1177/1751143717750788

Thomas, E. J. M. D, MPH; Sexton, J. Bryan PhD \& Helmreich R. L. P. (2003). Discrepant attitudes about teamwork among critical care nurses and physicians. Crit Care Med [Internet]. 31(3):956-9. 0.1097/01.CCM.0000056183.89175.76

Tlili M. A., Aouicha W., Ben Rejeb M., Sahli J., Ben Dhiab M., Chelbi S., Mtiraoui A., Laatiri H. S., Ajmi T., Zedini C., \& Mallouli M. (2020). Assessing patient safety culture in 18 Tunisian adult intensive care units and determination of its associated factors: A multi-center study. J Crit Care. 56:208-14. 10.1016/j.jcrc.2020.01.001

Tomazoni A., Rocha P. K., de Souza S., Anders J. C. \& de Malfussi H. F. C. (2014). Patient safety culture at neonatal intensive care units: Perspectives of the nursing and medical team. Rev Lat Am Enfermagem. 22(5):755-63. 10.1590/0104-1169.3624.2477

Tomazoni A., Rocha P. K., Ribeiro M. B., Serapião L. S., Souza S. \& Manzo B. F. (2017). Perception of nursing and medical professionals on patient safety in neonatal intensive care units | Segurança do paciente na percepção da enfermagem e medicina em unidades de terapia intensiva neonatal. Rev Gauch Enferm. 38(1):e64996. 10.1590/1983-1447.2017.01.64996

World Health Organization. (2019). Global action on patient safety Report by the Director-General. A72/26 [Internet]. Genebra. Available from: https://apps.who.int/iris/bitstream/handle/10665/328696/A72_26-en.pdf?sequence=1\&isAllowed=y 